\title{
Sarcomatoid Renal Cell Carcinoma: The Present and Future of Treatment Paradigms
}

\author{
Nellowe Candelario ${ }^{\mathrm{a}, \mathrm{b}}$, Christopher Geiger ${ }^{\mathrm{a}, \mathrm{b}}$ and Thomas Flaig ${ }^{\mathrm{a}, \mathrm{b}, *}$ \\ ${ }^{a}$ University of Colorado Cancer Center, University of Colorado Denver, Anschutz Medical Campus, Aurora, CO, \\ USA \\ ${ }^{\mathrm{b}}$ Division of Medical Oncology, School of Medicine, University of Colorado Denver, Anschutz Medical Campus, \\ Aurora, CO, USA
}

Received 1 July 2021

Accepted 8 October 2021

Pre-press 26 October 2021

Published 3 December 2021

\begin{abstract}
Sarcomatoid renal cell carcinoma (sRCC) is an aggressive form of kidney cancer that is associated with poor prognosis. It can arise from any histologic type of renal cell carcinoma. The majority of cases will present with advanced or metastatic disease requiring systemic therapy. Nephrectomy is the treatment of choice in locally resectable disease. The therapeutic options for sRCC have evolved in the past decade. Cytotoxic chemotherapy and monotherapy with targeted therapy (VEGF and mTOR) have historically shown poor response rates and survival in the treatment of metastatic sRCC. The use of checkpoint inhibitors and their combination with targeted therapy against VEGF has changed the landscape and outcomes for renal cell carcinoma. Given the rarity of sRCC most of the data on treatment is from small cohorts or extrapolation from larger clinical trials. The benefit from the combination of checkpoint inhibitors and targeted therapy to VEGF has shown promise in the sRCC population in post hoc analysis of large clinical trials. Future research focusing on further characterizing the unique biologic and clinical features of SRCC is critical in advancing the knowledge and developing effective therapy to improve clinical outcomes and survival.
\end{abstract}

\section{INTRODUCTION}

Kidney cancer is ranked as the 14th most common type of cancer worldwide. There are 76,080 estimated new cases of kidney and renal pelvis malignancies in the United States in 2021 representing $4 \%$ of all cancer cases. Approximately 13,780 people will die from kidney cancer in the United States in 2021 [1, 2]. Renal cell carcinoma (RCC) is classified based on morphologic, molecular, and genetic features. The most common type is clear cell which accounts for 70-90\% of cases. Other rare histologic types include the papillary and chromophobe types of renal cell carcinoma [3-5].

\footnotetext{
*Correspondence to: Thomas Flaig, MD., Mail Stop F520; 13001 East 17th Place; Aurora, CO 80045, USA. Tel.: +1 303 724 8155; E-mail: Thomas.Flaig@CUAnschutz.edu.
}

Renal cell carcinoma with sarcomatoid dedifferentiation (sRCC) is an aggressive form of kidney cancer that portends a poor prognosis [5,6]. Although it was historically classified as a distinct type of renal cell carcinoma, sRCC is now understood to be a dedifferentiation of renal carcinoma [6]. Sarcomatoid transformation can occur in any morphologic type of renal cell carcinoma. It is unknown if the primary histologic type undergoing sarcomatoid transformation affects prognosis. One series found the incidence of sarcomatoid differentiation was $8 \%$ in clear cell renal carcinoma, $3 \%$ in papillary renal carcinoma, $9 \%$ in chromophobe renal carcinoma, $29 \%$ in collecting duct carcinoma, and $11 \%$ in unclassified renal cell carcinoma [7]. Sarcomatoid renal cell carcinoma is characterized by a tumor composed of malignant spindle cells that have features of epithelial, stromal and myxoid components. The presence of sarcomatoid features in any type of renal cell carci- 
noma is classified as a poorly differentiated or high grade (Grade 4) disease [8-10]. Sarcomatoid dedifferentiation in RCC is an independent predictor of mortality regardless of stage of disease; in a stageby-stage analyses, sRCC patients have worse survival [11-13].

The treatment paradigm for renal cell carcinoma has evolved in the past 20 years particularly in the advanced and metastatic stages with the advent of immunotherapy (CTLA-4, PD-1 and PDL1 inhibitors) and therapy targeting the vascular endothelial growth factor (VEGF) pathway. This review aims to provide a perspective on the biology and current treatment strategies in the management of sRCC which can have implications for research development identifying additional targets of therapy.

\section{PATHOLOGIC FEATURES AND BIOLOGY}

Sarcomatoid renal cell carcinoma is characterized by pleomorphic spindle cells that resemble sarcoma [14]. The sarcomatoid dedifferentiation includes a variable amount of fibrosarcoma like or pleomorphic undifferentiated like patterns. In uncommon circumstances, features of osteosarcoma, chondrosarcoma or rhabdomyosarcoma can be appreciated. The presence of necrosis is almost universal [6,7]. The proportion of sarcomatoid features within the clinical entity of sRCC may vary; there is no consensus percentage of sarcomatoid component required to classify a renal cell carcinoma as having sarcomatoid dedifferentiation. A small proportion, approximately $3 \%$, of renal cell carcinoma, has been described as pure sarcomatoid [15]. Tumors with pure sarcomatoid features are identified as unclassified renal cell carcinoma (uRCC) given they do not have the distinctive features to be categorized further based on morphology. This is in contrast to RCC with $100 \%$ sarcomatoid features in which the histologic subtype can be identified [4, 5].

Although the classification of renal cell carcinoma as having sarcomatoid dedifferentiation does not require a minimum amount of sarcomatoid component on pathologic examination, the percentage of sarcomatoid features is clinically relevant $[7,15]$. It has been shown that with every $10 \%$ increase in the sarcomatoid component of the tumor, there is a $6 \%$ proportionate increase in mortality and that patients with tumors containing more than $30 \%$ sarcomatoid differentiation have a higher mortality [11]. While most sRCC patients present with advanced or metastatic disease, patients with early localized disease with more than $25 \%$ sarcomatoid features have higher mortality and recurrence rates $[12,13]$.

Histologically, immunohistochemistry markers associated with a renal origin of the tumor includes PAX2, CD10 and PAX8 are usually observed in sRCC and epithelial markers such as keratin, AE1/AE3, vimentin and epithelial membrane antigen (EMA) may also be positive. Carbonic anhydrase IX (CAIX), which is specific for clear cell renal cell carcinoma, can be appreciated in some cases of sRCC $[6,16,17]$. It is important to note that sarcomatoid transformation can lead to loss of renal cell carcinoma markers, particularly CAIX, CD10 and PAX8. Positive staining for CAIX is associated with poor prognosis in sRCC [18]. The key factor in establishing a diagnosis of sRCC is the morphologic sarcomatoid component within the tumor. Sarcomatoid renal cell carcinoma is conventionally classified as high grade or grade 4 $[7,15,16,19]$.

There are proposed mechanisms for the development of sarcomatoid dedifferentiation of renal cell carcinoma. It has been theorized that the two components of the tumor could come from two independent cells of origin or a single cell origin leading to both epithelial and sarcomatoid components (Fig. 1) [20, 21]. Genomic profiling to identify driver mutations in sRCC showed identical mutations in the sarcomatoid and epithelial component. TP53 (42.3\%), VHL (34.6\%), CDKN2A (26.9\%), and NF2 (19.2\%) were the most frequently altered genes [22]. This suggests that the origin of the sRCC possibly arises from a common progenitor with sRCC. Molecular characterization of renal cell carcinoma has shown that sRCC has lower PBRM1 and KDM5C expression, and these findings may be associated with increased angiogenesis. It is also notable that sarcomatoid dedifferentiated tumors have higher expression of PD-L1 and alteration in CDK2A/B [23]. A better understanding of the genomic drivers of sRCC may help to develop clinically actionable biomarkers in the future.

\section{CLINICAL PRESENTATION}

Sarcomatoid renal cell carcinoma is an aggressive tumor with a median survival of 9 months. In the non-metastatic setting, sRCC generally presents as a locally advanced tumor, with over $25 \%$ of those with localized disease presenting with a T4 lesion (tumor extending beyond the Gerota's fascia) and about one third with lymph node involvement [24, 


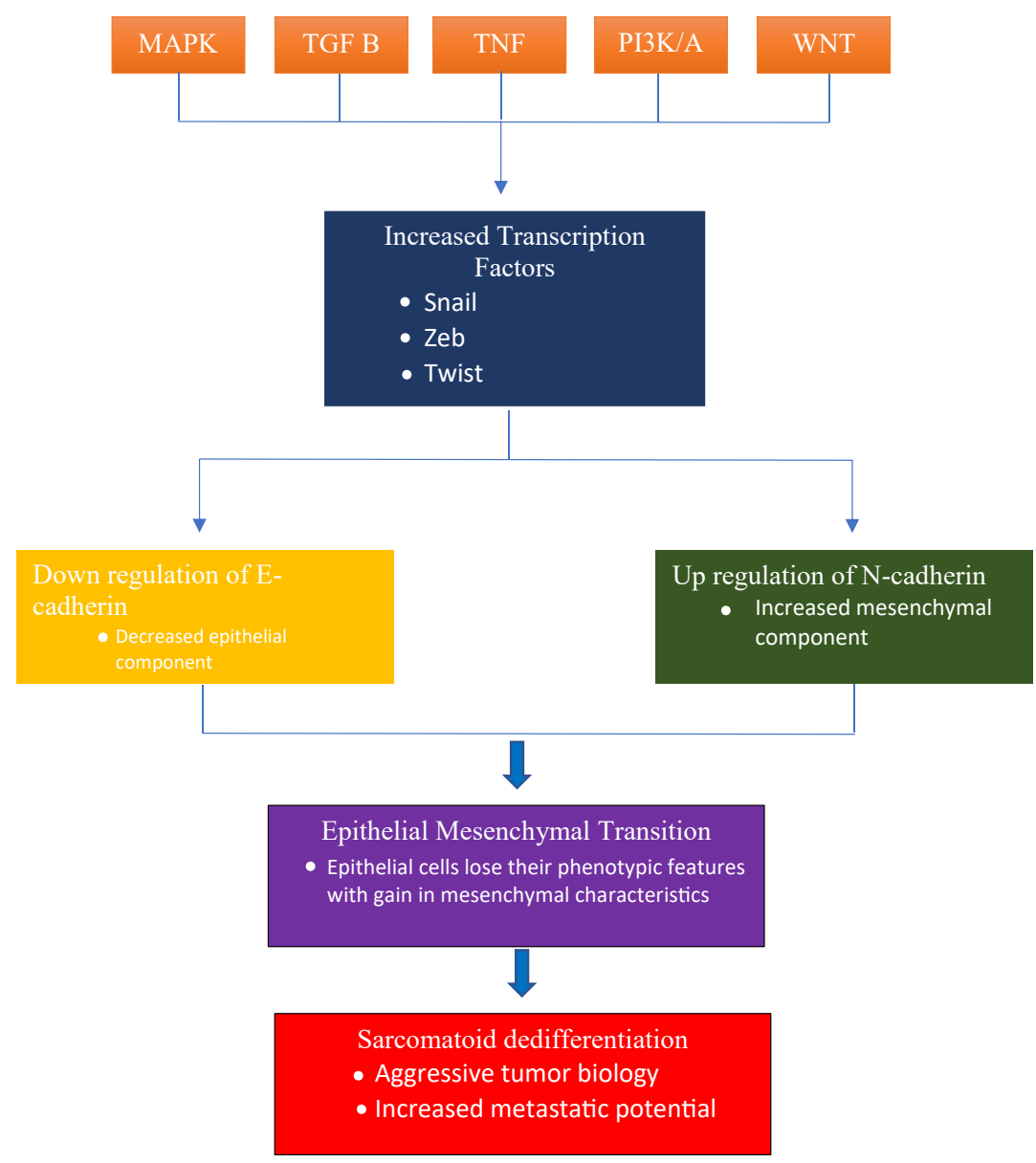

Fig. 1. Tumor Biology of Sarcomatoid Renal Cell Carcinoma: There are several pathways involved in the pathogenesis of sRCC. These include MAPK, TGF, TNF beta, PI3K/AK and WNT pathways. Signals from these pathways lead to amplification of Snail, Zab and Twist transcription factors. One of the central events in defining sRCC is an Epithelial Mesenchymal Transition (EMT). This happens due to a gain of mesenchymal component of the tumor characterized by upregulation of $\mathrm{N}$ - cadherin and loss of epithelial component from down regulation of E-cadherin. E-cadherin is a vital component of cell-to-cell adhesion to form tight junctions and its loss increases the propensity to metastasize, which is a key characteristic of the aggressive biology of sarcomatoid dedifferentiation of renal cell carcinoma [20].

25]. Unfortunately, the majority of sRCC cancers are metastatic on presentation. The most common distant metastatic sites include lungs $(67 \%)$, bone $(40 \%)$, liver $(21 \%)$, and brain $(15 \%)$ [26, 27]. The pattern of metastasis has prognostic implications in RCC including patients with sRCC. Thyroid, adrenal, bowel, lung, and pancreatic metastases appear to have a better prognosis compared to brain, bone and liver metastases [28, 29].

Presenting signs and symptoms of sRCC are nonspecific. The majority of patients will have abdominal or flank pain with or without hematuria. Patients with pulmonary metastasis can present with shortness of breath and cough. Constitutional symptoms are also seen, including fatigue, weight loss, fever, and night sweats $[20,24,26]$.

\section{TREATMENT}

\section{Surgical management}

Nephrectomy offers a potential cure for localized or early-stage sRCC. However, localized or early stage sRCC patients have poor outcomes even with optimal surgical management. A single center study of localized sRCC patients after nephrectomy found that the median time to recurrence of disease was 26 months [30]. The optimal surgical approach in localized sRCC is unclear. Some advocate for radical nephrectomy, although observational data suggest that partial nephrectomy may not be inferior in tumors less than $7 \mathrm{~cm}$ (T1 disease) [31]. An extensive lymphadenectomy at the time of surgery may improve 
outcomes in this high-risk population [32, 33]. Cytoreductive nephrectomy has been shown to improve survival among patients with metastatic renal cell carcinoma and is a standard of care for selected RCC patients [34]. A population-based study among patients with metastatic sRCC who underwent cytoreductive nephrectomy has shown that patients with $\mathrm{T} 1$ and $\mathrm{T} 2$ disease have a survival benefit of more than 6 months using this approach [35]. There has been a long-standing role of metastectomy among select patients with RCC who have oligometastatic disease. Patients with a disease-free interval greater than one year, younger than 60 years old, or solitary metastasis particularly in the lung may have the most benefit $[36,37]$. The role of metastectomy in sRCC is less clear and has only been reported in small cases series [38].

The role of adjuvant therapy in kidney cancer has been explored after nephrectomy. Sunitinib was compared to placebo in the S-TRAC trial in RCC with locoregional disease, which resulted in minimal improvement in disease free survival with increased toxicity; however, the number of sRCC patients was not reported in this trial [39]. Adjuvant pembrolizumab has been evaluated in RCC after nephrectomy and this effort included sRCC patients $(11 \%)$. The overall results showed a 2-year disease free survival of $77 \%$ in the treatment arm vs $68 \%$ in the placebo group [40].

\section{Role for radiation therapy}

Renal cell carcinoma is considered a radioresistant tumor using standard radiotherapy (RT) approaches [41, 42]. A meta-analysis evaluating post-operative radiation after nephrectomy in renal cell carcinoma has demonstrated improvement in locoregional failure. However, this did not translate to an overall survival benefit [43]. The role of RT in the nonmetastatic sRCC setting has been evaluated. Using SEER data for sRCC, adjuvant radiation therapy post nephrectomy did not improve disease specific or overall survival [44].

High doses of radiation have been classically utilized in brain and spinal metastasis in the form of stereotactic radiosurgery (SRS). SRS has also been used for radioresistant tumors like renal cell carcinoma presenting with brain metastasis demonstrating a $75 \%$ response rate [42]. This hypofractionated approach with higher doses of radiation for tumors outside of the central nervous system is known as stereotactic body radiotherapy (SBRT). The use of
SBRT has been evaluated in metastatic renal cell carcinoma and melanoma, both classically characterized as radioresistant tumors. An aggressive SBRT regimen of at least 45 Gy showed efficacy in achieving local control that is comparable to more radiosensitive cancer types [43]. Treatment schedule delivering up to 48 Gy in 3 to 4 fractions have been found to be effective and safe in renal cell carcinoma with little specific data on sRCC[45]. Therefore, the role of radiation therapy in either sarcomatoid or non-sarcomatoid RCC is largely limited to localized treatment of symptomatic metastatic disease particularly to the brain and bone but may be applied to soft tissue lesions as well $[44,46]$. The use of high dose of radiotherapy via SRS and SBRT appears to provide the most effective tumor control [42, 43].

\section{Systemic therapy}

Sarcomatoid renal cell carcinoma presenting with advanced or metastatic disease warrants the use of systemic therapy. These treatments include chemotherapy, targeted tyrosine kinase inhibitors, and immunotherapy. Given the rarity of sRCC and its exclusion from some clinicals, there are limited data on the most effective systemic therapy approaches. Most of the treatment guidance in SRCC is based on extrapolation of subgroup analyses from larger studies among the more common variants, particularly clear cell renal cell carcinoma. The choice of systemic therapy for advanced or metastatic renal cell carcinoma is guided by prognostic risk stratification models including the Memorial Sloan Kettering Cancer Center (MSKCC) and International Metastatic Renal cell carcinoma database consortium (IMDC) approaches. These risk stratification models classify typically classify patients with sRCC as intermediate or poor risk $[47,48]$.

\section{Cytotoxic chemotherapy}

Before the availability of targeted therapies and given the histologic resemblance of sRCC to sarcomas, cytotoxic chemotherapy has been evaluated in sRCC with the prospect of achieving the same responses (Table 1). In one retrospective study of fourteen sRCC patients treated with chemotherapy, 3 of the 8 patients receiving doxorubicin-based chemotherapy had a response, with an overall survival of 20, 29 and 60 months among patients who responded [49]. The combination of doxorubicin with ifosfamide, which is an active chemotherapy in soft 
Table 1

Chemotherapy studies in Sarcomatoid Renal Cell Carcinoma

\begin{tabular}{|c|c|c|c|c|c|c|}
\hline Study & Type of study & Treatment & $N$ & ORR & PFS/TTP & OS \\
\hline $\begin{array}{l}\text { Culine et al. } \\
\text { [49] }\end{array}$ & Retrospective & Doxorubicin based & 14 & $37.5 \%$ & $\mathrm{NR}$ & $20-60$ months \\
\hline $\begin{array}{l}\text { Escudier et al. } \\
\text { [50] }\end{array}$ & Phase 2 & Doxorubicin+Ifosfamide & 23 & $0 \%$ & 2.2 months & 3.9 months \\
\hline $\begin{array}{l}\text { Nanus et al. } \\
\text { [51] }\end{array}$ & Phase 2 & Gemcitabine+Doxorubicin & $18(10 \mathrm{sRCC})$ & $40 \%$ (sRCC) & $\begin{array}{l}5.2 \text { months } \\
\text { (sRCC) }\end{array}$ & NR \\
\hline $\begin{array}{l}\text { Haas et al. } \\
{[52]}\end{array}$ & Phase 2 & Gemcitabine+Doxorubicin & 39 & $16 \%$ & 3.5 months & 8.8 months \\
\hline
\end{tabular}

ORR: overall response rate; PFS: progression free survival; TTP: Time to progression; OS: overall survival; NR: Not reported.

tissue sarcoma, has been evaluated in a multicenter study of 25 sRCC patients. The findings of this study were disappointing with no objective responses and an overall survival of only 3.9 months [50]. The combination of gemcitabine and doxorubicin was also tested in two phase 2 studies [51, 52]. In the study by Nanus et al., 18 patients with RCC were treated with the combination of gemcitabine and doxorubicin. Ten patients were classified as sRCC and among these patients, only two (20\%) had a complete response while another 2 patients $(20 \%)$ had mixed or partial responses [51]. In the ECOG 8802 trial involving 39 sRCC patients, only six patients $(16 \%)$ of patients responded to gemcitabine and doxorubicin while about $26 \%$ had stable disease. Median progression free survival and overall survival were 3.5 months and 8.8 months respectively [52].

Treatment response to cytotoxic chemotherapy has some association with the proportion of sarcomatoid component. The ECOG 8802 trial showed that patients with $75 \%$ or greater sarcomatoid features have better response rates when treated with combination of gemcitabine and doxorubicin [52]. In a study evaluating the combination of gemcitabine and sunitinib, patients with more than $10 \%$ sarcomatoid features also had better treatment response and disease stability [53]. These data suggest that cytotoxic therapy may be a useful treatment option for some sRCC patients with higher proportions of sarcomatoid features on pathology.

In summary, cytotoxic chemotherapy generally has limited response rates and survival benefit. Unfortunately, the histologic similarities of sRCC to sarcomas has not translated to high response rates with chemotherapy. Although these studies were primarily limited by small number of subjects, single or combination chemotherapy is unlikely to yield durable clinically significant responses leading to survival benefit in sRCC.

\section{Targeted therapy (VEGF and MTOR inhibitors)}

Improved understanding of the biology of renal cell carcinoma has changed the spectrum of disease management and systemic therapy options in RCC. Targeted therapy directed against the vascular endothelial growth factor (VEGF) receptor and mammalian target of rapamycin (mTOR) pathway has been utilized as standard therapy for renal cell carcinoma patients [54-56]. Therapy directed against these pathways have been used as monotherapy or in combination with chemotherapy in sRCC (Table 2). Michaelson et al studied the combination of gemcitabine and sunitinib in sRCC, producing a response rate of $26 \%$ with greater responses among those tumors comprised of more than $10 \%$ sarcomatoid features [53]. A retrospective review evaluated the combination of gemcitabine, capecitabine and bevacizumab among sarcomatoid RCC and nonsarcomatoid RCC; over half of these patients had received prior tyrosine kinase inhibitors. Patients with SRCC in this analysis had poor PFS and OS at 3.9 months and 10.4 months respectively [57]. This combination was tested in a phase 2 trial of sRCC patients by Maiti et al., which showed a PFS of 5.5 months and OS of 12 months. The majority of patients (71\%) had disease progression during the trial [58]. The use of sorafenib after progression on chemotherapy with gemcitabine and doxorubicin was studied in a phase 2 clinical trial of sRCC patients and showed a low response rate at $11.1 \%$. The median time to progression seemed longer in this study at 10.9 months [59].

Large retrospective studies evaluating the use of various targeted therapies including multikinase inhibitors, mTOR and VEGF receptors blockers in sRCC show consistent PFS between 4 to 5 months and OS of 10 to 12 months compared to smaller prospective studies [60,61]. Temsirolimus and everolimus, inhibitors of mTOR, which is vital in the key pathways of tumorigenesis of renal cell carci- 
Table 2

Studies on Targeted therapies including combination therapy in Sarcomatoid Renal Cell Carcinoma

\begin{tabular}{|c|c|c|c|c|c|c|}
\hline Study & Type of study & Treatment & $N$ & ORR & PFS/ TTP & OS \\
\hline $\begin{array}{l}\text { Golshayan et al. } \\
\text { [60] }\end{array}$ & Retrospective & $\begin{array}{l}\text { Sunitinib; } \\
\text { Sorafenib; } \\
\text { Bevacizumab; Suni- } \\
\text { tinib + Bevacizumab }\end{array}$ & 43 & $19 \%$ & 5.3 months & 11.8 months \\
\hline Staehler et al. [59] & Phase 2 & $\begin{array}{l}\text { Sorafenib after } \\
\text { progression of disease } \\
\text { post gemcitabine and } \\
\text { doxorubicin }\end{array}$ & 9 & $11.1 \%$ & 10.9 months & NR \\
\hline Jonasch et al. [57] & Retrospective & $\begin{array}{l}\text { Gemcitabine }+ \\
\text { Capecitabine }+ \\
\text { Bevacizumab }\end{array}$ & $28(10 \mathrm{sRCC})$ & NR & $\begin{array}{l}3.9 \text { months } \\
\text { (sRCC) }\end{array}$ & 9 months (sRCC) \\
\hline Voss et al. [62] & Retrospective & $\begin{array}{l}\text { Temsirolimus; } \\
\text { Everolimus }\end{array}$ & 85 (23 sRCC) & $13 \%$ (sRCC) & $\begin{array}{l}3.5 \text { months } \\
\text { (sRCC) }\end{array}$ & $\begin{array}{l}8.2 \text { months } \\
\text { (sRCC) }\end{array}$ \\
\hline $\begin{array}{l}\text { Michaelson et al. } \\
\text { [53] }\end{array}$ & Phase 2 & $\begin{array}{l}\text { Gemcitabine + } \\
\text { Sunitinib }\end{array}$ & 39 & $26 \%$ & 5 months & 10 months \\
\hline $\begin{array}{l}\text { Kyriakopoulus et } \\
\text { al. [61] }\end{array}$ & Retrospective & $\begin{array}{l}\text { Sunitinib; Sorafenib; } \\
\text { Bevacizumab; } \\
\text { Temsirolimus; } \\
\text { Everolimus }\end{array}$ & 2,286 (230 sRCC) & $7 \%(\mathrm{sRCC})$ & $\begin{array}{l}4.5 \text { months } \\
\text { (sRCC) }\end{array}$ & $\begin{array}{l}10.4 \text { months } \\
\text { (sRCC) }\end{array}$ \\
\hline Maiti et al. [58] & Phase 2 & $\begin{array}{l}\text { Gemcitabine + } \\
\text { Capecitabine + } \\
\text { Bevacizumab }\end{array}$ & 34 & $20 \%$ & 5.5 months & 12 months \\
\hline
\end{tabular}

ORR: overall response rate; PFS: progression free survival; TTP: Time to progression; OS: overall survival; NR: Not reported.

noma, have also been evaluated in sRCC. In a study by Voss et al., patients with sRCC receiving mTOR inhibitors had a poor response rate of $13 \%$ with no complete responses and an overall survival of only 8 months [62].

Overall, the use targeted therapies alone or in combination with chemotherapy has demonstrated disappointing response rates, PFS and overall survival. There could be value using these medications sequentially as salvage therapy after progression to offer some disease stability as seen in the study of Staehler et al. [59].

\section{Immunotherapy}

Immunotherapy has been utilized in the management of renal cell carcinoma with interleukin-2 (IL-2) therapy approved in 1992. Interleukin-2 (IL-2) is a cytokine that modulates the immune system by promoting the expansion of cytotoxic and regulator T-cells to produce an anti-tumor effect [63]. High dose (HD) IL-2 has generated durable responses in a limited number of patients with good and poor risk metastatic renal cell carcinoma. Median overall survival was 43 months with this approach and some patients remained progression free for an extended period [64].

A study by Achkar et al., showed low response rates to HD IL-2 at $10 \%$, although the median over- all survival of 31 months among patients with sRCC appeared higher than historical controls [65]. In a retrospective study of 199 sRCC patients receiving cytokine or targeted therapy, $61 \%$ of patients who received cytokine therapy were alive at 1 year [66]. These findings highlight the potential role of HD IL-2 in the treatment of sRCC; however, the use of IL-2 has been limited by the toxicity profile and poor tolerance of this medication.

The integration of immune checkpoint inhibitors has changed the treatment landscape in the field of oncology including RCC. Cancer cells evade the immune system by activating different immune checkpoint pathways. Immune checkpoint inhibitors use monoclonal antibodies that target cytotoxic Tlymphocyte antigen 4 (CTLA-4), Program death-1 (PD-1) and Program Death-Ligand 1 (PD-L1). The effect of this monoclonal antibody inhibition is the activation and proliferation of effector T cells directed at cancer cells [67].

The CheckMate 214 trial compared the combination of ipilimumab/nivolumab to sunitinib among patients with intermediate and poor risk renal cell carcinoma. This study demonstrated excellent overall survival and response rate with the combination of ipilimumab and nivolumab compared to sunitinib [68]. A post hoc analysis among 74 patients with sRCC who received the immune checkpoint 
Table 3

Post hoc analysis of Phase III trials on Immunotherapy combination for Sarcomatoid Renal Cell Carcinoma

\begin{tabular}{|c|c|c|c|c|c|c|c|}
\hline Study & $\begin{array}{l}\text { Investigational } \\
\text { therapy }\end{array}$ & Comparator arm & $N(\%$ sRCC $)$ & ORR (CR) & 12-month OS & PFS & OS \\
\hline $\begin{array}{l}\text { CheckMate } 214 \\
\text { [69] }\end{array}$ & $\begin{array}{l}\text { Ipilimumab + } \\
\text { Nivolumab }\end{array}$ & Sunitinib & $74(18.2 \%)$ & $61 \%(19 \%)$ & $84 \%$ & 26.5 months & Not reached \\
\hline $\begin{array}{l}\text { KEYNOTE } 426 \\
{[72]}\end{array}$ & $\begin{array}{l}\text { Axitinib }+ \\
\text { Pembrolizumab }\end{array}$ & Sunitinib & $51(18.2 \%)$ & $59 \%(12 \%)$ & $83 \%$ & Not reached & Not reached \\
\hline $\begin{array}{l}\text { JAVELIN Renal } \\
101 \text { [75] }\end{array}$ & $\begin{array}{l}\text { Axitinib + } \\
\text { Avelumab }\end{array}$ & Sunitinib & $47(12.2 \%)$ & $47 \%(4 \%)$ & $83 \%$ & 7 months & NR \\
\hline $\begin{array}{l}\text { IMmotion } 151 \\
\text { [76] }\end{array}$ & $\begin{array}{l}\text { Atezolizumab + } \\
\text { Bevacizumab }\end{array}$ & Sunitinib & $68(16 \%)$ & $49 \%(10 \%)$ & $56 \%$ & 8.3 months & 21.7 months \\
\hline $\begin{array}{l}\text { Checkmate 9ER } \\
\text { [77] }\end{array}$ & $\begin{array}{l}\text { Nivolumab + } \\
\text { Cabozantinib }\end{array}$ & Sunitinib & $75(11.5 \%)$ & $\begin{array}{l}55.9 \%(\mathrm{CR} \\
\text { not reported })\end{array}$ & NR & 10.9 months & Not reached \\
\hline
\end{tabular}

ORR: Overall response rate; CR: Complete response; PFS: progression free survival; OS: overall survival; \%: percentage of overall population; NR: Not reported.

inhibitor combination showed an overall response rate of $61 \%(19 \% \mathrm{CR})$. Progression free survival was 26.5 months in sRCC patients receiving ipilimumab/nivolumab. Similar to the overall population, median OS was not reached in the study among sRCC patients [69].

There are several key studies that defined a role for immune checkpoint inhibitor therapy combined with anti-VEGF in the treatment of advanced sRCC. McGregor et al evaluated the use of the combination of atezolizumab and bevacizumab among patients with RCC with non-clear cell variant and RCC with more than $20 \%$ sarcomatoid features. This combination was found to be effective and safe with a response rate of 50\% among patients with SRCC [70]. The combination of axitinib and pembrolizumab was evaluated in the KEYNOTE 426 study. This combination showed improved response rates, median progression free survival and median overall survival over sunitinib [71]. Rini et al reported an exploratory analysis of the KEYNOTE 426 study among intermediate, poor risk RCC and those with sarcomatoid features. The results in the total population in this study were consistent for patients with sRCC. Overall response rate was 59\%. Both the median PFS and median OS were not reached among sRCC patients receiving the combination of axitinib and pembrolizumab [72]. The combination of immune checkpoint inhibitor and anti-angiogenic agents has been examined in two other large phase III studies $[73,74]$. Post hoc analysis among patients with sRCC showed improved response rates over $45 \%$ and median PFS greater than 6 months [75, 76]. The CheckMate 9ER trial compared the combination of cabozantinib plus nivolumab to sunitinib. In the subset of patients with sRCC, the overall response rate was $55.9 \%$ with a median PFS of
10.9 months [77]. The CLEAR study evaluated the combination of lenvatinib and pembrolizumab in advanced RCC, which also included sRCC (11.8\%). In the overall population, median PFS was significantly longer at 23.9 months with an overall response rate as high as $71 \%$ using the combination therapy. This yielded some of the highest response rates among all the TKI+immunotherapy combinations to date in RCC patients. A post-hoc analysis in the sRCC group for the CLEAR study is not yet available but the overall findings of this combination are encouraging [78]. A phase 1 study evaluating the use of the combination of ipilimumab, cabozantinib and nivolumab (ICONIC trial) in rare metastatic genitourinary malignancies included sRCC patients with at least $50 \%$ sarcomatoid features and showed an overall response rate of $62.5 \%$ in this subset [79]. Taken together, it is clear from these trials that patients with sRCC benefit from the combination of immunotherapy and anti-angiogenic therapy.

These large prospective phase III trials have shown improved outcomes in the treatment of sRCC (Table 3). The objective response rates ranged from $47 \%$ to $61 \%$, which also resulted in improved PFS and OS [69, 72, 75-77]. The benefit among these patients could be explained by increased PD-L1 expression and higher levels of tumor infiltrating lymphocytes (TILs) in sRCC [23, 80, 81]. It is important to highlight that despite improved outcomes of immunotherapy-based treatment in sRCC, both PFS and $\mathrm{OS}$ are still inferior compared to non-sarcomatoid counterparts [82].

Genomic and biomarker analysis with the combination of immune checkpoint inhibitor and antiangiogenic therapy has shown improved outcomes in tumors with high immunogenic or anti-tumor 


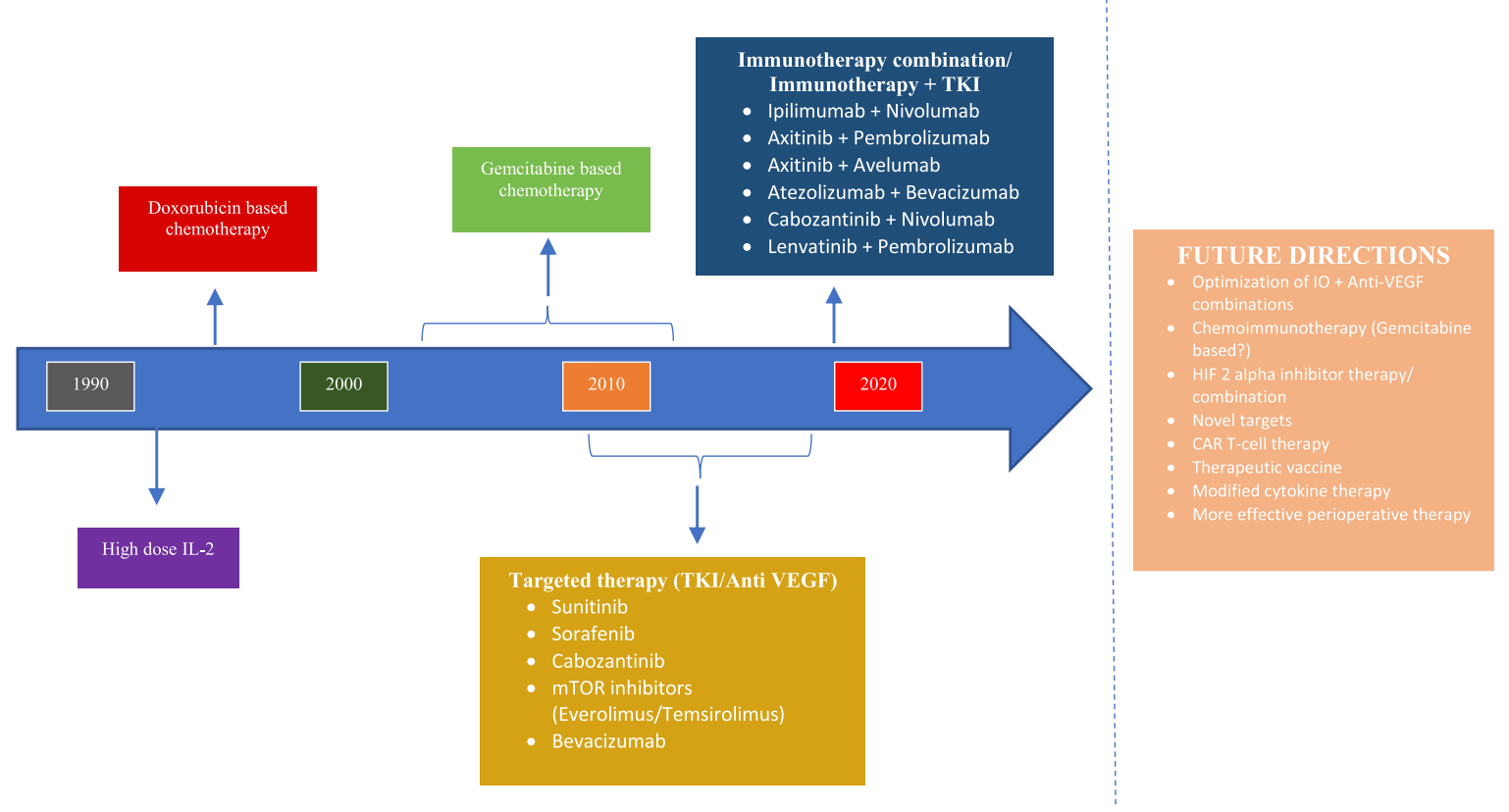

Fig. 2. Evolution of the treatment paradigm for Metastatic Sarcomatoid Renal Cell Carcinoma.

immunity gene profile. A low angiogenic gene signature is also predictive of response to therapy. The PD-L1 positivity and tumor mutational burden markers have not consistently predicted benefit in these large trials [83-85].

\section{OVERALL TREATMENT APPROACH}

Among patients with localized and resectable disease, nephrectomy still provides the best opportunity for cure although recurrence rates are high. Lymphadenectomy at the time of nephrectomy has been advocated among patients with sarcomatoid features given that approximately $38 \%$ of sRCC patients with high-risk features demonstrate pathologic nodal involvement at the time of surgery [32, 33]. Post nephrectomy finding of sarcomatoid features greater than $25 \%$ in the primary tumor portends worse outcomes and vigilant follow up is needed for these patients [12]. The use of pembrolizumab in the adjuvant setting may be considered in SRCC patients with data supporting improved disease-free survival [40]. The potential benefit of cytoreductive nephrectomy appears to correlate with the amount of sarcomatoid component present in the tumor and may be more beneficial in patients with $\mathrm{T} 1$ and $\mathrm{T} 2$ disease $[35,86]$. Radiation therapy in the form of
SRS or SBRT is an option for local disease control in patients with symptomatic metastatic disease [42-44, 46].

The majority of sRCC patients will have metastatic disease requiring the use of systemic therapy (Fig. 2). Immune checkpoint inhibitor combination or immunotherapy in combination with VEGF inhibitors have shown some of the most promising response rates and survival benefit in metastatic sRCC to date [69, 72, 75-78]. Although these data are from post hoc analyses and extrapolation from larger trials, this approach offers the most compelling preferred systemic treatment for sRCC currently. The data from the CheckMate 214 study with the use of the combination of nivolumab and ipilimumab also show very notable overall survival outcomes in the intermediate and poor risk patients with a median OS of 48.1 months in the overall population. Based on available data, this combination also has compelling complete and overall response rates in patient with sRCC $[69,87]$. The data on the combination of lenvatinib and pembrolizumab has generated among the highest overall response rate reported in the overall population at $71 \%$. The response among sRCC has not been reported [80]. Selecting the appropriate combination has generally been based on the patient's underlying medical condition, toxicity concerns, and prognostic risk. 
There are generally limited response rates and overall outcomes with the use of cytotoxic chemotherapy or targeted therapy alone [49-52]. It is important to note that in select patients with a higher proportion of sarcomatoid features there may be a benefit from gemcitabine-based chemotherapy [51, 52]. Patients with a significant proportion of sarcomatoid features, a large burden of disease, and those who need rapid responses may be candidates for chemotherapy alone or in combination with targeted therapies when there is a contraindication to immune checkpoint inhibitors. In these cases, anti VEGF TKI chemotherapy-based regimen could be considered.

High dose IL-2 has shown some utility with response rates and survival benefit possibly better than monotherapy with targeted therapy or chemotherapy for SRCC. Although the use of HD IL2 has limited application due to poor tolerance, this is an option for treatment among patients who are physically fit. The use of targeted therapy as a monotherapy has also shown suboptimal response and survival benefit [53, 57-62]. Combination targeted therapy and cytotoxic therapy could be considered as second line treatment for sRCC.

\section{FUTURE DIRECTIONS IN SARCOMATOID RENAL CELL CARCINOMA}

The treatment approach to metastatic RCC has changed dramatically over the last 15 years, with many new treatment options. It is important to better define which patients with sarcomatoid features will benefit from specific therapeutic options, including cytotoxic chemotherapy, targeted therapy, or immunotherapy, in order to further improve outcomes.

A promising treatment in renal cell carcinoma is the use of Hypoxia inducible factor (HIF) 2 alpha inhibitors, knowing the vital role of the von HippelLindau protein (pVHL) in the transcription of HIF and tumorigenesis in renal cell carcinoma. HIF-2 alpha inhibitors have been developed among VHL associated tumors including RCC [88]. Belzutifan, an oral HIF-2 alpha inhibitor, has been evaluated in VHL associated RCC, and showed overall response rate of $49 \%$ in this population with a median duration of response not yet reached with 20.2 months of follow up [89]. This therapy was approved for use in cancers associated with VHL in August of 2021. Sarcomatoid renal cell carcinoma arising from clear cell renal cell carcinoma is known to have a higher expression of HIF [90]. The use of HIF-2 alpha inhibition alone or in combination with existing therapeutic options for sRCC including immunotherapy is an exciting avenue for future research.

The use of chemoimmunotherapy with the combination of chemotherapy and checkpoint inhibitors have been used in a variety of malignancies including lung, GI, and urothelial malignancies. Given the historical responses of sRCC to gemcitabine-based chemotherapy, this could be a potential basis of chemoimmunotherapy for sRCC in future investigations.

The success of immunotherapy and VEGF inhibitor combination in this setting provides a backbone for further combination therapy in the treatment of RCC. Precision immunotherapy with the use of monoclonal antibodies, adaptive cell therapy, and therapeutic vaccines, either alone or in combination, are approaches that have the potential to advance therapy further in the near future for sRCC [81].

Improving outcomes with adjuvant therapy is important across renal cell carcinoma. Trials implementing anti-angiogenic therapy with VEGF inhibitors in the post nephrectomy setting have been disappointing $[39,91]$. The KEYNOTE- 564 study has shown benefit with the use of pembrolizumab in the adjuvant setting post-nephrectomy [40]. This study has provided a basis for further adjuvant studies using immunotherapy alone or in combination. Given the worse prognosis of localized sRCC, developing effective adjuvant treatment is paramount.

Chimeric T- Cell therapy (CAR-T cell) has seen successful in various hematologic malignancies. This is a form of immunotherapy modulation to directly attack cancer cells. Studies using CAR-T cell therapy in solid tumors include renal cell carcinoma [92, 93]. Understanding the distinct molecular biology of sRCC and its response to immunotherapy, CAR- T cell therapy is a potential therapy.

While treatment options in renal cell carcinoma have evolved over the last decade, sarcomatoid renal cell carcinoma has inferior outcomes compared to non-sarcomatoid RCC. Ongoing research to improve outcomes in this population should focus on future efforts to understand the biology and discover therapeutic options improve survival among these patients. Inclusion of sRCC in clinical trials in a meaningful way, either as a subset of larger trials or in stand-alone trials, is essential to advancing our understanding. 


\section{CONCLUSION}

Sarcomatoid renal carcinoma is an aggressive tumor with high morbidity and mortality. It is believed to arise from an epithelial mesenchymal transition, giving the tumor a high metastatic potential. Patients with localized and resectable tumors should undergo a radical nephrectomy which offers a potential cure of the disease. Unfortunately, recurrence rates are high even with optimal surgical management in patients with localized disease. The role of immunotherapy and other targeted therapy in the adjuvant setting is a potential area for future investigation to improve post nephrectomy survival.

Most patients with sRCC will present with metastatic disease. The use of immune checkpoint inhibitors has changed landscape of treatment of renal cell carcinoma overall. The use of combination of anti-angiogenic medications with immunotherapy has translated to clinically meaningful improvement in response rate on overall survival in RCC patients, with the strong suggestion of this benefit extending to sRCC based on the post hoc analysis of larger trials. A dedicated research effort to further characterize the unique biologic and clinical features of sRCC is critical to advance our understanding and improve outcomes through better therapy.

\section{ACKNOWLEDGMENTS}

The authors have no acknowledgments.

\section{FUNDING}

The authors report no funding.

\section{AUTHOR CONTRIBUTIONS}

NC: performed literature search, wrote and edited manuscript; CG: editing and critical revision of the manuscript; TF: oversaw, edited and critical revision of manuscript. All authors have read and approved the final manuscript for submission

\section{CONFLICT OF INTEREST}

NC has no relevant disclosure; CG has no relevant disclosure, TF: Has received research/clinical trial funding from Novartis, Dendreon, GTX, Janssen, Medivation, Sanofi, BMS, Roche/Genentech, Exelixis, Aragon, Sotio, Tokai, AZ/MedImmune, Lilly, Settle Genetics, La Roche-Posay, Merck, Seagen.
He has provided consulting for Janssen and Seattle Genetics.

$\mathrm{TF}$ is an Editorial Board member of this journal, but was not involved in the peer-review process of this manuscript nor had access to any information regarding its peer-review.

\section{REFERENCES}

[1] World Health Organization International Agency for Research on Cancer (IARC). GLOBOCAN 2020: estimated cancer incidence, mortality and prevalence worldwide in 2020. June 1, 2021]; Available from: https://gco.iarc. fr/today/data/factsheets/cancers/29-Kidney-fact-sheet.pdf.

[2] Surveillance, Epidemiology, and End Results (SEER) Program SEER*Stat Database: Incidence - SEER Research Data, 9 Registries, Nov 2020 Sub (1975-2018) - Linked To County Attributes - Time Dependent (1990-2018) Income/Rurality, 1969-2019 Counties, National Cancer Institute, DCCPS, Surveillance Research Program.

[3] Moch H., et al., The 2016 WHO Classification of Tumours of the Urinary System and Male Genital Organs-Part A: Renal, Penile, and Testicular Tumours. Eur Urol. 2016; 70(1):93-105.

[4] Warren AY, Harrison D. WHO/ISUP classification, grading and pathological staging of renal cell carcinoma: standards and controversies. World Journal of Urology. 2018;36(12):1913-1926.

[5] Delahunt B, et al., The International Society of Urological Pathology (ISUP) Grading System for Renal Cell Carcinoma and Other Prognostic Parameters. American Journal of Surgical Pathology 2013;37(10):1490-1504.

[6] Mouallem NE, Smith SC, Paul AK. Sarcomatoid renal cell carcinoma: Biology and treatment advances. Urologic Oncology: Seminars and Original Investigations. 2018;36(6):265-271.

[7] de Peralta-Venturina M, et al., Sarcomatoid differentiation in renal cell carcinoma: a study of 101 cases. Am J Surg Pathol. 2001;25(3):275-84.

[8] Delahunt B. Sarcomatoid renal carcinoma: the final common dedifferentiation pathway of renal epithelial malignancies. Pathology. 1999;31(3):185-190.

[9] Delahunt B, et al., Grading of renal cell carcinoma. Histopathology. 2018;74(1):4-17.

[10] National Comprehensive Cancer Network. Kidney Cancer (Version 4.2011) [cited 2021 June 1, 2021]; Available from: https://www.nccn.org/professionals/physician_gls/pdf/kidn ey.pdf.

[11] Zhang BY, et al., A novel prognostic model for patients with sarcomatoid renal cell carcinoma. BJU International. 2014;115(3):405-411.

[12] Kim T, et al., Using percentage of sarcomatoid differentiation as a prognostic factor in renal cell carcinoma. Clinical Genitourinary Cancer. 2015;13(3):225-230.

[13] Trudeau V, et al., Comparison of oncologic outcomes between sarcomatoid and clear cell renal cell carcinoma. World Journal of Urology. 2016;34(10):1429-1436.

[14] Farrow GM, Harrison EG, Utz DC. Sarcomas and sarcomatoid and mixed malignant tumors of the kidney in adults—part III. Cancer. 1968;22(3):556-563.

[15] Wei S, Al-Saleem T. The pathology and molecular genetics of sarcomatoid renal cell carcinoma: a mini-review. Journal of Kidney Cancer and VHL. 2017;4(2):19-23. 
[16] Kuroda N, et al., Review of sarcomatoid renal cell carcinoma with focus on clinical and pathobiological aspects. Histol Histopathol. 2003;18(2):551-5.

[17] DeLong W, et al., Sarcomatoid renal cell carcinoma. An immunohistochemical study of 18 cases. Arch Pathol Lab Med. 1993;117(6):636-40.

[18] Yu W, et al., Distinct immunophenotypes and prognostic factors in renal cell carcinoma with sarcomatoid differentiation: a systematic study of 19 immunohistochemical markers in 42 cases. BMC Cancer. 2017;17(1): 293-293.

[19] Cheville JC, et al., Sarcomatoid Renal Cell Carcinoma. The American Journal of Surgical Pathology. 2004;28(4): 435-441.

[20] Blum KA, et al., Sarcomatoid renal cell carcinoma: biology, natural history and management. Nature Reviews. Urology. 2020;17(12):659-678.

[21] Anani W, et al., A series of collision tumors in the genitourinary tract with a review of the literature. Pathology Research and Practice. 2014;210(4):217-223.

[22] Malouf GG, et al., Genomic characterization of renal cell carcinoma with sarcomatoid dedifferentiation pinpoints recurrent genomic alterations. European Urology. 2016;70(2):348-357.

[23] Motzer RJ, et al., Molecular subsets in renal cancer determine outcome to checkpoint and angiogenesis blockade. Cancer Cell. 2020;38(6):803-817.e4.

[24] Mian BM, et al., Prognostic factors and survival of patients with sarcomatoid renal cell carcinoma. Journal of Urology. 2002;167(1):65-70.

[25] Goekkurt E, et al., Pharmacogenetic analyses of a phase III trial in metastatic gastroesophageal adenocarcinoma with fluorouracil and leucovorin plus either oxaliplatin or cisplatin: a study of the arbeitsgemeinschaft internistische onkologie. J Clin Oncol. 2009;27(17):2863-73.

[26] Shuch B, et al., Sarcomatoid renal cell carcinoma: a comprehensive review of the biology and current treatment strategies. The Oncologist. 2012;17(1):46-54.

[27] Cangiano T, et al., Sarcomatoid renal cell carcinoma: biologic behavior, prognosis, and response to combined surgical resection and immunotherapy. Journal of Clinical Oncology. 1999;17(2):523-523.

[28] Gong J, et al., Metastasis in renal cell carcinoma: Biology and implications for therapy. Asian J Urol. 2016;3(4):286292.

[29] Dudani S, et al., Evaluation of clear cell, papillary, and chromophobe renal cell carcinoma metastasis sites and association with survival. JAMA Network Open. 2021;4(1):e2021869-e2021869.

[30] Merrill MM, et al., Clinically nonmetastatic renal cell carcinoma with sarcomatoid dedifferentiation: Natural history and outcomes after surgical resection with curative intent. Urologic Oncology. 2015;33(4):166.e21-166.e1.66E29.

[31] Ji B, et al., Propensity-score matched comparison of partial versus radical nephrectomy for T1N0M0 sarcomatoid renal cell carcinoma. Translational Andrology and Urology. 2020;9(2):250-257.

[32] Crispen PL, et al., Lymph node dissection at the time of radical nephrectomy for high-risk clear cell renal cell carcinoma: indications and recommendations for surgical templates. European Urology. 2011;59(1):18-23.

[33] Capitanio U, et al., Lymph node dissection in renal cell carcinoma. European Urology. 2011;60(6):1212-1220.

[34] Flanigan RC, et al., Nephrectomy followed by interferon Alfa-2b compared with interferon Alfa-2b alone for metastatic renal-cell cancer. New England Journal of Medicine. 2001;345(23):1655-1659.

[35] Ji B, et al., A population study to identify candidates for cytoreductive nephrectomy in patients with metastatic sarcomatoid renal cell carcinoma from the surveillance, epidemiology, and end results (SEER) database. Medical Science Monitor : International Medical Journal of Experimental and Clinical Research. 2020;26:e921297-e921297.

[36] Zhao Y, et al., Prognostic factors for overall survival after lung metastasectomy in renal cell cancer patients: A systematic review and meta-analysis. International Journal of Surgery. 2017;41:70-77.

[37] Lee-Ying R, Lester R, Heng DYC. Current management and future perspectives of metastatic renal cell carcinoma. International Journal of Urology. 2014;21(9):847-855.

[38] Ueno T. et al., Pulmonary metastasectomy from renal cell carcinoma including 3 cases with sarcomatoid component. General Thoracic and Cardiovascular Surgery. 2016;64(3):149-152.

[39] Ravaud A, et al., Adjuvant sunitinib in high-risk renalcell carcinoma after nephrectomy. New England Journal of Medicine. 2016;375(23):2246-2254.

[40] Choueiri TK, et al., Adjuvant pembrolizumab after nephrectomy in renal-cell carcinoma. New England Journal of Medicine. 2021;385(8):683-694.

[41] Siva S, et al., Radiotherapy for renal cell carcinoma: renaissance of an overlooked approach. Nature Reviews Urology 2017;14(9):549-563.

[42] Sayan M, et al., Evaluation of response to stereotactic radiosurgery in patients with radioresistant brain metastases. Radiation Oncology Journal. 2019;37(4):265-270.

[43] Stinauer MA, et al., Stereotactic body radiation therapy for melanoma and renal cell carcinoma: impact of single fraction equivalent dose on local control. Radiation Oncology (London, England). 2011;6:34-34.

[44] Dabestani S, et al., Local treatments for metastases of renal cell carcinoma: a systematic review. The Lancet Oncology. 2014;15(12):e549-e561.

[45] Francolini G, et al., Stereotactic body radiation therapy (SBRT) on renal cell carcinoma, an overview of technical aspects, biological rationale and current literature. Critical Reviews in Oncology/Hematology, 2018;131:24-29.

[46] Ljungberg B, et al., European association of urology guidelines on renal cell carcinoma: The 2019 Update. European Urology. 2019;75(5):799-810.

[47] Motzer RJ, et al., Interferon-Alfa as a comparative treatment for clinical trials of new therapies against advanced renal cell carcinoma. Journal of Clinical Oncology. 2002;20(1):289296.

[48] Heng DY, et al., Prognostic factors for overall survival (OS) in patients with metastatic renal cell carcinoma (RCC) treated with vascular endothelial growth factor (VEGF)targeted agents: Results from a large multicenter study. Journal of Clinical Oncology. 2009;27(15_suppl):50415041.

[49] Culine S, et al., Treatment of sarcomatoid renal cell carcinoma: is there a role for chemotherapy? European Urology. 1995;27(2):138-141.

[50] Escudier B, et al., Doxorubicin and ifosfamide in patients with metastatic sarcomatoid renal cell carcinoma: a Phase II study of the genitourinary group of the french federation of cancer centers. Journal of Urology. 2002;168(3):959-961.

[51] Nanus DM, et al., Active chemotherapy for sarcomatoid and rapidly progressing renal cell carcinoma. Cancer. 2004;101(7):1545-1551. 
[52] Haas NB, et al., A phase II trial of doxorubicin and gemcitabine in renal cell carcinoma with sarcomatoid features: ECOG 8802. Medical oncology (Northwood, London, England). 2012;29(2):761-767.

[53] Jay R, et al., Phase 2 trial of sunitinib and gemcitabine in patients with sarcomatoid and/or poor-risk metastatic renal cell carcinoma. Michaelson MD, McKay RR, Werner L, Atkins MB, Van Allen EM, Olivier KM, Song J, Signoretti S, McDermott DF, Choueiri TK.Cancer. 2015 Oct 1;121(19):3435-43. [Epub 2015 Jun 8]. doi: 10.1002/cncr.29503. Urologic Oncology: Seminars and Original Investigations. 2017;35(3):117-118.

[54] Stitzlein L, Rao PSS, Dudley R. Emerging oral VEGF inhibitors for the treatment of renal cell carcinoma. Expert Opinion on Investigational Drugs. 2018;28(2):121-130.

[55] Escudier B, et al., Sorafenib in advanced clear-cell renal-cell carcinoma. New England Journal of Medicine. 2007;356(2):125-134.

[56] Motzer RJ, et al., Sunitinib versus interferon alfa in metastatic renal-cell carcinoma. New England Journal of Medicine. 2007;356(2):115-124.

[57] Jonasch E, et al., Treatment of metastatic renal carcinoma patients with the combination of gemcitabine, capecitabine and bevacizumab at a tertiary cancer centre. BJU International. 2011;107(5):741-747.

[58] Maiti A, et al., Phase 2 trial of capecitabine, gemcitabine, and bevacizumab in sarcomatoid renal-cell carcinoma. Clinical Genitourinary Cancer. 2017:S1558-7673(17) 30238-0.

[59] Staehler M, et al., Sorafenib after combination therapy with gemcitabine plus doxorubicine in patients with sarcomatoid renal cell carcinoma: a prospective evaluation. European Journal of Medical Research. 2010;15(7):287-291.

[60] Golshayan AR, et al., Metastatic sarcomatoid renal cell carcinoma treated with vascular endothelial growth factor-targeted therapy. Journal of Clinical Oncology. 2009;27(2):235-241.

[61] Kyriakopoulos CE, et al., Outcome of patients with metastatic sarcomatoid renal cell carcinoma: results from the international metastatic renal cell carcinoma database consortium. Clinical Genitourinary Cancer. 2015;13(2):e79-e85.

[62] Voss MH, et al., Treatment outcome with mTOR inhibitors for metastatic renal cell carcinoma with nonclear and sarcomatoid histologies. Annals of Oncology : Official Journal of the European Society for Medical Oncology. 2014;25(3):663-668.

[63] Fyfe G, et al., Results of treatment of 255 patients with metastatic renal cell carcinoma who received highdose recombinant interleukin-2 therapy. Journal of Clinical Oncology. 1995;13(3):688-696.

[64] McDermott DF, et al., The high-dose aldesleukin "select" trial: a trial to prospectively validate predictive models of response to treatment in patients with metastatic renal cell carcinoma. Clinical Cancer Research : An Official Journal of the American Association for Cancer Research. 2015;21(3):561-568.

[65] Achkar T, et al., High-dose interleukin 2 in patients with metastatic renal cell carcinoma with sarcomatoid features. PloS One. 2017;12(12):e0190084-e0190084.

[66] Keskin SK, et al., Outcomes of patients with renal cell carcinoma and sarcomatoid dedifferentiation treated with nephrectomy and systemic therapies: comparison between the cytokine and targeted therapy Eras. The Journal of Urology. 2017;198(3):530-537.
[67] Darvin P, et al., Immune checkpoint inhibitors: recent progress and potential biomarkers. Experimental \& Molecular Medicine. 2018;50(12):1-11.

[68] Motzer RJ, et al., Nivolumab plus ipilimumab versus sunitinib in advanced renal-cell carcinoma. The New England Journal of Medicine. 2018;378(14):1277-1290.

[69] Tannir NM, et al., Efficacy and safety of nivolumab plus ipilimumab versus sunitinib in first-line treatment of patients with advanced sarcomatoid renal cell carcinoma. Clinical Cancer Research. 2020;27(1):78-86.

[70] McGregor BA, et al., Results of a multicenter phase ii study of Atezolizumab and Bevacizumab for patients with metastatic renal cell carcinoma with variant histology and/or sarcomatoid features. Journal of Clinical Oncology : Official Journal of the American Society of Clinical Oncology. 2020;38(1):63-70.

[71] Rini BI, et al., Pembrolizumab plus axitinib versus sunitinib for advanced renal-cell carcinoma. $\mathrm{N}$ Engl $\mathrm{J}$ Med. 2019;380(12):1116-1127.

[72] Rini BI, et al., Pembrolizumab (pembro) plus axitinib (axi) versus sunitinib as first-line therapy for metastatic renal cell carcinoma (mRCC): Outcomes in the combined IMDC intermediate/poor risk and sarcomatoid subgroups of the phase 3 KEYNOTE-426 study. Journal of Clinical Oncology. 2019;37(15_suppl):4500-4500.

[73] Motzer RJ, et al., Aavelumab plus axitinib versus sunitinib for advanced renal-cell carcinoma. N Engl J Med. 2019;380(12):1103-1115.

[74] Rini BI, et al., Atezolizumab plus bevacizumab versus sunitinib in patients with previously untreated metastatic renal cell carcinoma (IMmotion151): a multicentre, openlabel, phase 3, randomised controlled trial. Lancet. 2019;393(10189):2404-2415.

[75] Choueiri TK, et al., Efficacy and biomarker analysis of patients (pts) with advanced renal cell carcinoma (aRCC) with sarcomatoid histology (sRCC): Subgroup analysis from the phase III JAVELIN renal 101 trial of first-line avelumab plus axitinib (A+Ax) vs sunitinib (S). Annals of Oncology. 2019;30:v361.

[76] Rini BI, et al., Atezolizumab plus bevacizumab versus sunitinib for patients with untreated metastatic renal cell carcinoma and sarcomatoid features: a prespecified subgroup analysis of the immotion151 clinical trial. European Urology. 2021;79(5):659-662.

[77] Motzer RJ, et al., Nivolumab+cabozantinib (NIVO+CABO) versus sunitinib (SUN) for advanced renal cell carcinoma (aRCC): Outcomes by sarcomatoid histology and updated trial results with extended follow-up of CheckMate 9ER. Journal of Clinical Oncology. 2021;39(6_suppl): 308-308.

[78] Motzer R, et al., Lenvatinib plus pembrolizumab or everolimus for advanced renal cell carcinoma. New England Journal of Medicine. 2021;384(14):1289-1300.

[79] Apolo $A B$, et al., Final results from a phase I trial and expansion cohorts of cabozantinib and nivolumab (CaboNivo) alone or with ipilimumab (CaboNivoIpi) for metastatic genitourinary tumors. Journal of Clinical Oncology. 2021;39(6_suppl):3-3.

[80] Debien V, et al., Sarcomatoid dedifferentiation in renal cell carcinoma: from novel molecular insights to new clinical opportunities. Cancers. 2019;12(1):99.

[81] Braun DA, et al., Beyond conventional immune-checkpoint inhibition - novel immunotherapies for renal cell carcinoma. Nature reviews. Clinical Oncology. 2021;18(4): 199-214. 
[82] Kwak C, et al., Sarcomatoid differentiation as a prognostic factor for immunotherapy in metastatic renal cell carcinoma. Journal of Surgical Oncology. 2007;95(4):317-323.

[83] Motzer RJ, et al., Avelumab plus axitinib versus sunitinib in advanced renal cell carcinoma: biomarker analysis of the phase 3 JAVELIN Renal 101 trial. Nature Medicine. 2020;26(11):1733-1741.

[84] McDermott DF, et al., Clinical activity and molecular correlates of response to atezolizumab alone or in combination with bevacizumab versus sunitinib in renal cell carcinoma. Nature Medicine. 2018;24(6):749-757.

[85] Motzer RJ, et al., Biomarker analyses from the phase III CheckMate 214 trial of nivolumab plus ipilimumab $(\mathrm{N}+\mathrm{I})$ or sunitinib (S) in advanced renal cell carcinoma (aRCC). Journal of Clinical Oncology. 2020;38(15_suppl):5009-5009.

[86] Shuch B, et al., Cytoreductive nephrectomy for kidney cancer with sarcomatoid histology-is up-front resection indicated and, if not, is it avoidable? The Journal of Urology. 2009; 182(5):2164-2171.

[87] Albiges L, et al., Nivolumab plus ipilimumab versus sunitinib for first-line treatment of advanced renal cell carcinoma: extended 4-year follow-up of the phase III CheckMate 214 trial. ESMO Open. 2020;5(6):e001079.

[88] Choueiri TK, Kaelin WG. Targeting the HIF2-VEGF axis in renal cell carcinoma. Nature Medicine. 2020;26(10): 1519-1530.
[89] Srinivasan R, et al., Phase 2 study of belzutifan (MK-6482), an oral hypoxia-inducible factor $2 \alpha$ (HIF- $2 \alpha$ ) inhibitor, for Von Hippel-Lindau (VHL) disease-associated clear cell renal cell carcinoma (ccRCC). Journal of Clinical Oncology. 2021;39(15_suppl):4555-4555.

[90] Tickoo SK, et al., Immunohistochemical expression of hypoxia inducible factor- $1 \alpha$ and its downstream molecules in sarcomatoid renal cell carcinoma. Journal of Urology. 2007;177(4):1258-1263.

[91] Sun M, et al., Adjuvant vascular endothelial growth factortargeted therapy in renal cell carcinoma: a systematic review and pooled analysis. European Urology. 2018;74(5):611620.

[92] Lamers $\mathrm{CH}$, et al., Treatment of metastatic renal cell carcinoma with CAIX CAR-engineered T cells: clinical evaluation and management of on-target toxicity. Molecular Therapy: the Journal of the American Society of Gene Therapy. 2013;21(4):904-912.

[93] Lamers Cor HJ, et al., Treatment of metastatic renal cell carcinoma (mRCC) with CAIX CAR-engineered T-cells-a completed study overview. Biochemical Society Transactions. 2016;44(3):951-959. 